\title{
Robust Adaptive Fuzzy Control of Chaos in the Permanent Magnet Synchronous Motor
}

\author{
Jinpeng Yu, ${ }^{1}$ Junwei Gao, ${ }^{1,2}$ Yumei Ma, ${ }^{1}$ Haisheng Yu, ${ }^{1}$ \\ and Songfeng $\operatorname{Pan}^{1}$ \\ ${ }^{1}$ Institute of Complexity Science, Qingdao University, Qingdao 266071, China \\ ${ }^{2}$ State Key Laboratory of Rail Traffic Control and Safety, Beijing Jiaotong University, Beijing 100044, China \\ Correspondence should be addressed to Jinpeng Yu, yjp1109@hotmail.com
}

Received 5 May 2010; Accepted 12 July 2010

Academic Editor: Recai Kilic

Copyright (C) 2010 Jinpeng Yu et al. This is an open access article distributed under the Creative Commons Attribution License, which permits unrestricted use, distribution, and reproduction in any medium, provided the original work is properly cited.

\begin{abstract}
An adaptive fuzzy control method is developed to control chaos in the permanent magnet synchronous motor drive system via backstepping. Fuzzy logic systems are used to approximate unknown nonlinearities, and an adaptive backstepping technique is employed to construct controllers. The proposed controller can suppress the chaos of PMSM and track the reference signal successfully. The simulation results illustrate its effectiveness.
\end{abstract}

\section{Introduction}

Permanent magnet synchronous motors (PMSMs) are intensively used in industrial applications due to their high speed, high efficiency, high power density, and large torque to inertia ratio. Then, it is still a challenging problem to control the PMSM to get the perfect dynamic performance, because the dynamic model of PMSM is nonlinear, multivariable and even experiencing Hopf bifurcation, limit cycles, and chaotic attractors with systemic parameters falling into a certain area [1]. The chaotic behavior in PMSM is undesirable since it can extremely destroy the stabilization of the motor or even induce drive system collapse. Chaos in the PMSM and its control have been an active research area in the field of nonlinear control of electric motors [2]. Up to now, some control methods, such as OGY method [3], feedback linearization [4], time delay feedback control [5-7], sliding model control [8], adaptive control method [9, 10], backstepping method [11-14], and dynamic surface control [9] are successfully used to control or suppress chaos in PMSM. However, the existing control methods also have some disadvantages. The OGY method requires a variable system parameter which is usually unavailable in the control of the PMSM. The employed method of feedback linearization requires the exact mathematical model; so the controller 
requires the desired dynamics to replace the system at the $d-q$ axis stator currents. The time delay feedback control was successfully implemented to control the PMSM, but it is difficult to determine the time delay for TDFC method given a special target and is not suitable when the desired target is not the equilibrium or an unstable periodic orbit of the system. Chattering phenomenon and high heat loss in electrical power circuits are the drawbacks of the sliding mode control.

Backstepping is a newly developed technique to control the nonlinear systems with parameter uncertainty, particularly those systems in which the uncertainty does not satisfy matching conditions. Though the conventional backstepping is successfully applied to the control of PMSM drivers recently, it usually makes the designed controllers' structure to be very complex.

In recent years, fuzzy logic control (FLC) [15-17] has been found one of the most popular and conventional tools in functional approximations. An FLC has strong ability of handling uncertain information and can be easily used in the control of systems which is ill-defined or too complex to have a mathematical model. The essential part of an FLC is a set of the linguistic control rules related by the dual concepts of fuzzy implication and the compositional rule of inference [18]. Classically, fuzzy variables have been adjusted by expert knowledge and trial and error. It provides an effective way to design control system that is one of the important applications in the area of control engineering.

In this paper, an adaptive fuzzy control method is developed to control chaos in the permanent magnet synchronous motor drive systems via backstepping technology. During the controller design process, fuzzy logic systems are employed to approximate the nonlinearities of the chaotic PMSM drive system; the adaptive fuzzy controllers are constructed via backstepping. Compared with the conventional backstepping, the designed fuzzy controller has a simple structure, which can suppress the chaos of PMSM and track the reference signal generated by a reference model quite well.

\section{Mathematical Model of Chaotic PMSM Drive System and Preliminaries}

The dimensionless mathematical model of PMSM with the smooth air gap can be described as follows [1]:

$$
\begin{aligned}
& \frac{d \omega}{d t}=\sigma\left(i_{q}-\omega\right)-\tilde{T}_{L}, \\
& \frac{d i_{q}}{d t}=-i_{q}-i_{d} \omega+\gamma \omega+\tilde{u}_{q}, \\
& \frac{d i_{d}}{d t}=-i_{d}+i_{q} \omega+\tilde{u}_{d},
\end{aligned}
$$

where $\omega, i_{d}$ and $i_{q}$ are state variables, which denote angle speed and the $d-q$ axis currents, respectively. $\sigma$ and $\gamma$ are system operating parameters, which are positive. $\widetilde{T}_{L}, \tilde{u}_{d}$, and $\tilde{u}_{q}$ stand for the $d-q$ axis voltages and load torque, respectively. 


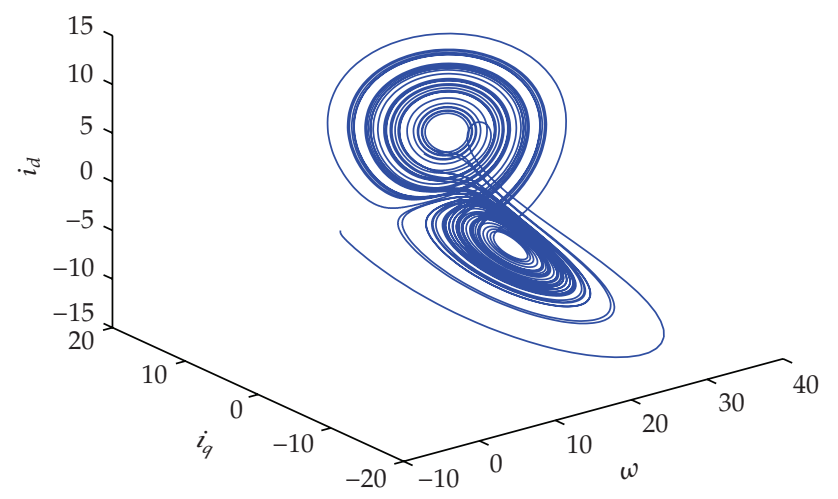

Figure 1: Typical chaotic attractor in PMSM with system parameters $\sigma=5.45$ and $\gamma=20$.

In system (2.1), the external inputs are set to zero, namely, $\widetilde{T}_{L}=\tilde{u}_{d}=\tilde{u}_{q}=0[1]$. Then, the system (2.1) becomes an unforced system:

$$
\begin{aligned}
& \frac{d \omega}{d t}=\sigma\left(i_{q}-\omega\right), \\
& \frac{d i_{q}}{d t}=-i_{q}-i_{d} \omega+\gamma \omega, \\
& \frac{d i_{d}}{d t}=-i_{d}+i_{q} \omega .
\end{aligned}
$$

The modern nonlinear theory such as bifurcation and chaos has been widely used to study the stability of PMSM driver system. The study found that the PMSM is experiencing chaotic behavior when the operating parameters $\sigma$ and $\gamma$ fall into a certain regime. For example, the PMSM displays chaos with $\sigma=5.45$ and $\gamma=20$. The typical chaotic attractor is shown in Figure 1. These chaotic oscillations can destroy the stabilization of the PMSM drive system. In order to remove or control chaos, we use $u_{d}$ as the manipulated variable which is desirable for the real application. Then, an adaptive fuzzy control approach is proposed to control chaos in the PMSM drive system via the backstepping technique. For simplicity, the following notations are introduced: $x_{1}=\omega, x_{2}=i_{q}$, and $x_{3}=i_{d}$. By using these notations, the dynamic model of PMSM driver system can be described by the following differential equations:

$$
\begin{aligned}
& \dot{x}_{1}=\sigma\left(x_{2}-x_{1}\right), \\
& \dot{x}_{2}=-x_{2}-x_{1} x_{3}+\gamma x_{1}, \\
& \dot{x}_{3}=-x_{3}+x_{1} x_{2}+u_{d} .
\end{aligned}
$$

The control objective is to design an adaptive fuzzy controller such that the state variable $x_{1}$ follows the given reference signal $x_{d}$, and all the closed-loop signals are bounded. 
To this end, we adopt the singleton fuzzifier, product inference, and the center-defuzzifier to deduce the following fuzzy rules:

$$
R_{i}: \text { IF } x_{1} \text { is } F_{1}^{i} \text { and } \cdots \text { and } x_{n} \text { is } F_{n}^{i} \text { THEN } y \text { is } B^{i} \quad(i=1,2, \ldots, N) \text {, }
$$

where $x=\left[x_{1}, \ldots, x_{n}\right]^{T} \in R^{n}$ and $y \in R$ are the input and output of the fuzzy system, respectively $F_{i}^{j}$ and $B^{i}$ are fuzzy sets in $R$. The fuzzy inference engine performs a mapping from fuzzy sets in $R^{n}$ to fuzzy set in $R$ based on the IF-THEN rules in the fuzzy rule base and the compositional rule of inference. The fuzzifier maps a crisp point $x=\left[x_{1}, \ldots, x_{n}\right]^{T} \in R^{n}$ into a fuzzy set $A_{x}$ in $R$. The defuzzifier maps a fuzzy set in $R$ to a crisp point in $R$. Since the strategy of singleton fuzzification, center-average defuzzification, and product inference is used, the output of the fuzzy system can be formulated as

$$
y(x)=\frac{\sum_{j=1}^{N} W_{j} \prod_{i=1}^{n} \mu_{F_{i}^{j}}\left(x_{i}\right)}{\sum_{j=1}^{N}\left[\prod_{i=1}^{n} \mu_{F_{i}^{j}}\left(x_{i}\right)\right]},
$$

where $W_{j}$ is the point at which fuzzy membership function $\mu_{B^{j}}\left(W_{j}\right)$ achieves its maximum value, and it is further assumed that $\mu_{B^{j}}\left(W_{j}\right)=1$. Let $p_{j}(x)=\prod_{i=1}^{n} \mu_{F_{i}^{j}}\left(x_{i}\right) / \sum_{j=1}^{N}\left[\prod_{i=1}^{n} \mu_{F_{i}^{j}}\left(x_{i}\right)\right]$, $S(x)=\left[p_{1}(x), p_{2}(x), \ldots, p_{N}(x)\right]^{T}$, and $W=\left[W_{1}, \ldots, W_{N}\right]^{T}$, then the fuzzy logic system above can be rewritten as

$$
y(x)=W^{T} S(x)
$$

If all memberships are taken as Gaussian functions, then the following lemma holds.

Lemma 2.1 (see [19]). Let $f(x)$ be a continuous function defined on a compact set $\Omega$. Then for any scalar $\varepsilon>0$, there exists a fuzzy logic system in the form of (2.6) such that

$$
\sup _{x \in \Omega}|f(x)-y(x)| \leq \varepsilon .
$$

\section{Adaptive Fuzzy Controller with the Backstepping Technique}

In this section, we will develop an adaptive fuzzy control approach to control chaos in PMSM drive system via the backstepping. The backstepping design procedure contains 3 steps. At each design step, a virtual control function $\alpha_{i}(i=1,2)$ will be constructed by using an appropriate Lyapunov function. At the last step, the real controller is constructed to control the system.

Step 1. For the reference signal $x_{d}$, define the tracking error variable as $z_{1}=x_{1}-x_{d}$. From the first differential equation of (2.3), the error dynamic system is given by $\dot{z}_{1}=\sigma\left(x_{2}-x_{1}\right)-\dot{x}_{d}$. 
Choose Lyapunov function candidate as $V_{1}=(1 / 2) z_{1}^{2}$, then the time derivative of $V_{1}$ is computed by

$$
\dot{V}_{1}=z_{1} \dot{z}_{1}=z_{1} \sigma\left(x_{2}-x_{1}-\frac{\dot{x}_{d}}{\sigma}\right)
$$

Construct the virtual control law $\alpha_{1}$ as

$$
\alpha_{1}\left(x_{1}, x_{d}, \dot{x}_{d}\right)=-\bar{k}_{1} z_{1}+x_{1}+\frac{\dot{x}_{d}}{\sigma}
$$

where $\bar{k}_{1}$ is a positive constant. By using (3.2), (3.1) can be rewritten in the following form:

$$
\dot{V}_{1}=-\bar{k}_{1} \sigma z_{1}^{2}+\sigma z_{1} z_{2}=-k_{1} z_{1}^{2}+\sigma z_{1} z_{2}
$$

with $k_{1}>0$ being a design parameter and $z_{2}=x_{2}-\alpha_{1}$.

Step 2. Differentiating $z_{2}$ gives

$$
\dot{z}_{2}=\dot{x}_{2}-\dot{\alpha}_{1}=-x_{2}-x_{1} x_{3}+\gamma x_{1}-\dot{\alpha}_{1}
$$

Now, choose the Lyapunov function candidate as $V_{2}=V_{1}+(1 / 2) z_{2}^{2}$. Obviously, the time derivative of $V_{2}$ is given by

$$
\dot{V}_{2}=\dot{V}_{1}+z_{2} \dot{z}_{2}=-k_{1} z_{1}^{2}+z_{2}\left(\sigma z_{1}-x_{2}-x_{1} x_{3}+\gamma x_{1}-\dot{\alpha}_{1}\right) .
$$

In the realistic model of PMSM, limited to the work conditions, the parameter $\gamma$ is unknown. So it cannot be used to construct the control signal. Thus, let $\hat{\gamma}$ be the estimation of $\gamma$. The corresponding adaptation laws will be specified later. The virtual control $\alpha_{2}$ is constructed as

$$
\alpha_{2}\left(Z_{2}\right)=-\frac{1}{x_{1}}\left(-k_{2} z_{2}-\sigma z_{1}+x_{2}-\hat{\gamma} x_{1}+\dot{\alpha}_{1}\right)
$$

where $k_{2}>0$ is a positive design parameter and $Z_{2}=\left[x_{1}, x_{2}, x_{d}, \dot{x}_{d}, \ddot{x}_{d}, \widehat{\gamma}\right]^{T}$. Adding and subtracting $\alpha_{2}$ in the bracket in (3.5) shows that

$$
\dot{V}_{2}=-k_{1} z_{1}^{2}-k_{2} z_{2}^{2}-x_{1} z_{2} z_{3}-z_{2}(\widehat{\gamma}-\gamma) x_{1}
$$

with $z_{3}=x_{3}-\alpha_{2}$.

Step 3. Differentiating $z_{3}$ results in the following differential equation:

$$
\dot{z}_{3}=\dot{x}_{3}-\dot{\alpha}_{2}=-x_{3}+x_{1} x_{2}+u_{d}-\dot{\alpha}_{2} .
$$


Choose the Lyapunov function candidate as $V_{3}=V_{2}+(1 / 2) z_{3}^{2}$. Furthermore, differentiating $V_{3}$ yields

$$
\begin{aligned}
\dot{V}_{3} & =\dot{V}_{2}+z_{3} \dot{z}_{3}=\dot{V}_{2}+z_{3}\left(-x_{3}+x_{1} x_{2}+u_{d}-\dot{\alpha}_{2}\right) \\
& =-k_{1} z_{1}^{2}-k_{2} z_{2}^{2}-z_{2}(\widehat{\gamma}-\gamma) x_{1}+z_{3}\left(f_{3}+u_{d}\right),
\end{aligned}
$$

where

$$
\begin{aligned}
\dot{\alpha}_{1} & =\dot{x}_{1}-\dot{x}_{d}=\sigma\left(x_{2}-x_{1}\right)-\dot{x}_{d} \\
\dot{\alpha}_{2} & =\sum_{i=1}^{2} \frac{\partial \alpha_{2}}{\partial x_{i}} \dot{x}_{i}+\sum_{i=0}^{2} \frac{\partial \alpha_{2}}{\partial x_{d}^{(i)}} x_{d}^{(i+1)}+\frac{\partial \alpha_{2}}{\partial \widehat{\gamma}} \dot{\hat{\gamma}} \\
& =\frac{\partial \alpha_{2}}{\partial x_{1}} \sigma\left(x_{2}-x_{1}\right)+\frac{\partial \alpha_{2}}{\partial x_{2}}\left(-x_{2}-x_{1} x_{3}+\gamma x_{1}\right)+\sum_{i=0}^{2} \frac{\partial \alpha_{2}}{\partial x_{d}^{(i)}} x_{d}^{(i+1)}+\frac{\partial \alpha_{2}}{\partial \widehat{\gamma}} \\
f_{3}(Z) & =-x_{3}+x_{1} x_{2}-x_{1} z_{2}-\dot{\alpha}_{2} \\
Z & =\left[x_{1}, x_{2}, x_{3}, x_{d}, \dot{x}_{d}, \ddot{x}_{d}, \widehat{\gamma}\right]^{T} .
\end{aligned}
$$

Notice that $f_{3}$ containing the derivative of $\alpha_{2}$, therewithal, the unknown parameter $\gamma$ appears in the expression of $f_{3}$. This will make the classical adaptive backstepping design become very complex and troubled, and the designed control law $u_{d}$ will have a complex structure. To avoid this trouble and simplify the control signal structure, we will employ the fuzzy logic system to approximate the nonlinear function $f_{3}$. As shown later, the design procedure of $u_{d}$ becomes simple, and $u_{d}$ is of a simple structure.

According to Lemma 2.1, for any given $\varepsilon_{3}>0$, there exists a fuzzy logic system $W_{3}^{T} S(Z)$ such that

$$
f_{3}(Z)=W_{3}^{T} S(Z)+\delta_{3}(Z)
$$

where $\delta_{3}(Z)$ is the approximation error and satisfies $\left|\delta_{3}\right| \leq \varepsilon_{3}$. Consequently, a straightforward calculation produces the following inequality:

$$
z_{3} f_{3}(Z)=z_{3}\left(W_{3}^{T} S(Z)+\delta_{3}(Z)\right) \leq \frac{1}{2 l_{3}^{2}} z_{3}^{2}\left\|W_{3}\right\|^{2} S^{2}+\frac{1}{2} l_{3}^{2}+\frac{1}{2} z_{3}^{2}+\frac{1}{2} \varepsilon_{3}^{2}
$$

Thus, it follows immediately from substituting (3.6) into (3.9) that

$$
\dot{V}_{3} \leq-k_{1} z_{1}^{2}-k_{2} z_{2}^{2}-z_{2}(\widehat{\gamma}-\gamma) x_{1}+\frac{1}{2 l_{3}^{2}} z_{3}^{2}\left\|W_{3}\right\|^{2} S^{2}+\frac{1}{2} l_{3}^{2}+\frac{1}{2} z_{3}^{2}+\frac{1}{2} \varepsilon_{3}^{2}+z_{3} u_{d}
$$


At this present stage, the control law $u_{d}$ is designed as

$$
u_{d}=-k_{3} z_{3}-\frac{1}{2} z_{3}-\frac{1}{2 l_{3}^{2}} z_{3} \widehat{\theta} S^{2}
$$

where $\widehat{\theta}$ is the estimation of the unknown constant $\theta$ which will be specified later. Define $\theta=\left\|W_{3}\right\|^{2}$. Furthermore, using equality (3.14), it can be verified easily that

$$
\dot{V}_{3} \leq-\sum_{i=1}^{3} k_{i} z_{i}^{2}+\frac{1}{2 l_{3}^{2}} z_{3}^{2}\left(\left\|W_{3}\right\|^{2}-\widehat{\theta}\right) S^{T}(Z) S(Z)+\frac{1}{2} l_{3}^{2}+\frac{1}{2} \varepsilon_{3}^{2}+z_{2}(\gamma-\widehat{\gamma}) x_{1} .
$$

Introduce variables $\tilde{\gamma}$ and $\tilde{\theta}$ as

$$
\begin{aligned}
& \tilde{r}=\widehat{r}-r, \\
& \tilde{\theta}=\widehat{\theta}-\theta,
\end{aligned}
$$

and choose the Lyapunov function candidate as

$$
V=V_{3}+\frac{1}{2 r_{1}} \tilde{\gamma}^{2}+\frac{1}{2 r_{2}} \tilde{\theta}^{2}
$$

where $r_{i}, i=1,2$ are positive constants. By differentiating $V$ and taking (3.15)-(3.17) into account, one has

$$
\begin{aligned}
\dot{V} & \leq-\sum_{i=1}^{3} k_{i} z_{i}^{2}+\frac{1}{2 l_{3}^{2}} z_{3}^{2} \tilde{\theta} S^{T}(Z) S(Z)+z_{2}(\gamma-\widehat{\gamma}) x_{1}+\frac{1}{2} l_{3}^{2}+\frac{1}{2} \varepsilon_{3}^{2}+\frac{1}{r_{1}} \tilde{\gamma} \dot{\hat{\gamma}}+\frac{1}{r_{2}} \tilde{\theta} \dot{\hat{\theta}} \\
& =-\sum_{i=1}^{3} k_{i} z_{i}^{2}+\frac{1}{2} l_{3}^{2}+\frac{1}{2} \varepsilon_{3}^{2}+\frac{1}{r_{1}} \tilde{\gamma}\left(-r_{1} z_{2} x_{1}+\dot{\hat{\gamma}}\right)+\frac{1}{r_{2}} \tilde{\theta}\left[-\frac{1}{2 l_{3}^{2}} z_{3}^{2} S^{T}(Z) S(Z)+\dot{\hat{\theta}}\right] .
\end{aligned}
$$

According to (3.18), the corresponding adaptive laws are chosen as follows:

$$
\begin{aligned}
& \dot{\hat{\gamma}}=r_{1} z_{2} \dot{\alpha}_{1}-m_{1} \widehat{\gamma}, \\
& \dot{\hat{\theta}}=\frac{1}{2 l_{3}^{2}} z_{3}^{2} S^{T}(Z) S(Z)-m_{2} \widehat{\theta},
\end{aligned}
$$

where $m_{i}$, for $i=1,2$ and $l_{3}$ are positive constants. 


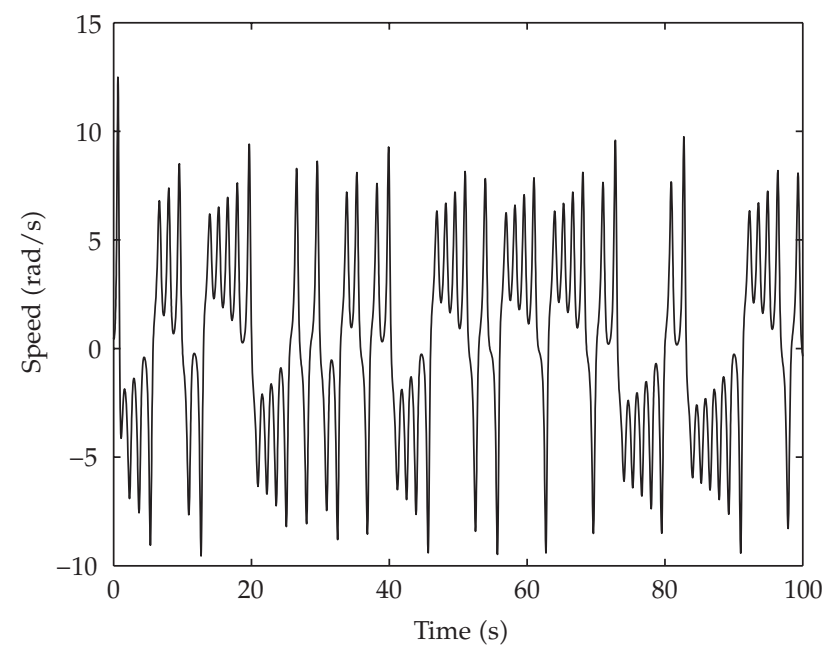

Figure 2: The $\omega$ curve of the chaotic PMSM drive system without $u_{d}$.

\section{Stability Analysis}

To address the stability analysis of the resulting closed-loop system, substitute (3.19) into (3.18) to obtain that

$$
\dot{V} \leq-\sum_{i=1}^{3} k_{i} z_{i}^{2}+\frac{1}{2} l_{3}^{2}+\frac{1}{2} \varepsilon_{3}^{2}-\frac{m_{1}}{r_{1}} \tilde{\gamma} \widehat{\gamma}-\frac{m_{2}}{r_{2}} \tilde{\theta} \widehat{\theta}
$$

For the term $-\tilde{\gamma} \hat{\gamma}$, one has $-\tilde{\gamma} \hat{\gamma} \leq-\tilde{\gamma}(\tilde{\gamma}+\gamma) \leq-(1 / 2) \tilde{\gamma}^{2}+(1 / 2) \gamma^{2}$. Similarly, $-\tilde{\theta} \widehat{\theta} \leq$ $-(1 / 2) \tilde{\theta}^{2}+(1 / 2) \theta^{2}$ holds. Consequently, by using these inequalities, (4.1) can be rewritten in the following form:

$$
\begin{aligned}
\dot{V} & \leq-\sum_{i=1}^{3} k_{i} z_{i}^{2}-\frac{m_{1}}{2 r_{1}} \widetilde{\gamma}^{2}-\frac{m_{2}}{2 r_{2}} \tilde{\theta}^{2}+\frac{1}{2} l_{3}^{2}+\frac{1}{2} \varepsilon_{3}^{2}+\frac{m_{1}}{2 r_{1}} r^{2}+\frac{m_{2}}{2 r_{2}} \theta^{2} \\
& \leq-a_{0} V+b_{0}
\end{aligned}
$$

where $a_{0}=\min \left\{2 k_{1}, 2 k_{2}, 2 k_{3}, m_{1}, m_{2}\right\}$ and $b_{0}=(1 / 2) l_{3}^{2}+(1 / 2) \varepsilon_{3}^{2}+\left(m_{1} / 2 r_{1}\right) r^{2}+\left(m_{2} / 2 r_{2}\right) \theta^{2}$. Furthermore, (4.2) implies that

$$
V(t) \leq\left(V\left(t_{0}\right)-\frac{b_{0}}{a_{0}}\right) e^{-a_{0}\left(t-t_{0}\right)}+\frac{b_{0}}{a_{0}} \leq V\left(t_{0}\right)+\frac{b_{0}}{a_{0}}, \quad \forall t \geqslant t_{0} .
$$




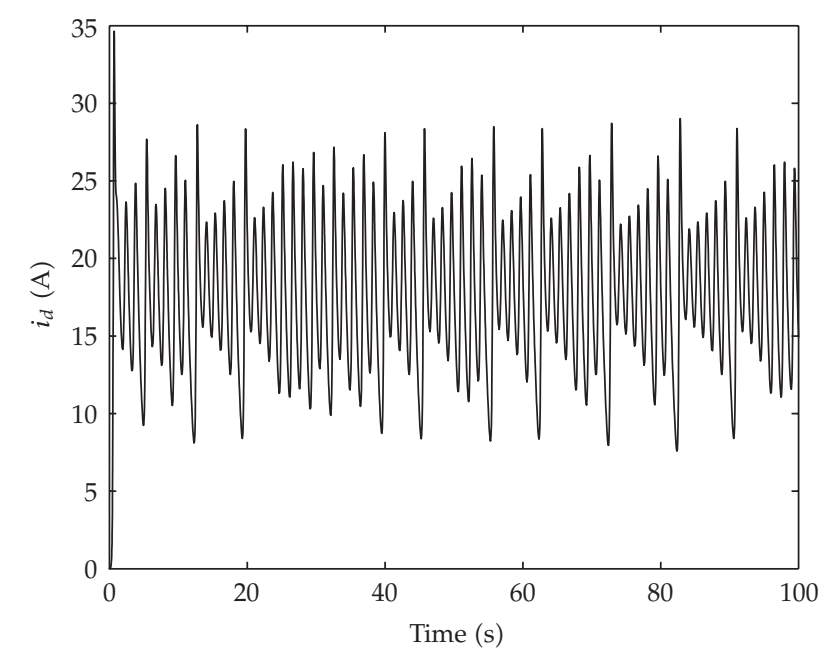

Figure 3: The $i_{d}$ curve of the chaotic PMSM drive system without $u_{d}$.

As a result, all $z_{i}(i=1,2,3), \tilde{\gamma}$, and $\tilde{\theta}$ belong to the compact set

$$
\Omega=\left\{\left(z_{i}, \tilde{\gamma}, \tilde{\theta}\right) \mid V \leq V\left(t_{0}\right)+\frac{b_{0}}{a_{0}}, \forall t \geqslant t_{0}\right\} .
$$

Namely, all the signals in the closed-loop system are bounded. Especially, from (4.3) we have

$$
\lim _{t \rightarrow \infty} z_{1}^{2} \leq \frac{2 b_{0}}{a_{0}}
$$

From the definitions of $a_{0}$ and $b_{0}$, it is clear that to get a small tracking error by taking $r_{i}$ sufficiently large and $l_{i}$ and $\varepsilon_{i}$ small enough after giving the parameters $k_{i}$ and $m_{i}$.

\section{Simulation}

In order to illustrate the effectiveness of the proposed results, the simulation will be conducted to control chaos in PMSM drive system with two sets. First we tested the chaotic PMSM drive system with $u_{d}=0$, which are shown in Figures 2, 3, and 4 . Then the proposed adaptive fuzzy approach in this paper is used to control the chaotic PMSM system, which are shown in Figures 5, 6, 7, and 8. The control parameters are chosen as follows:

$$
k_{1}=2, \quad k_{2}=20, \quad k_{3}=15, \quad r_{1}=r_{2}=15, \quad m_{1}=m_{2}=0.005, \quad l_{3}=0.2
$$




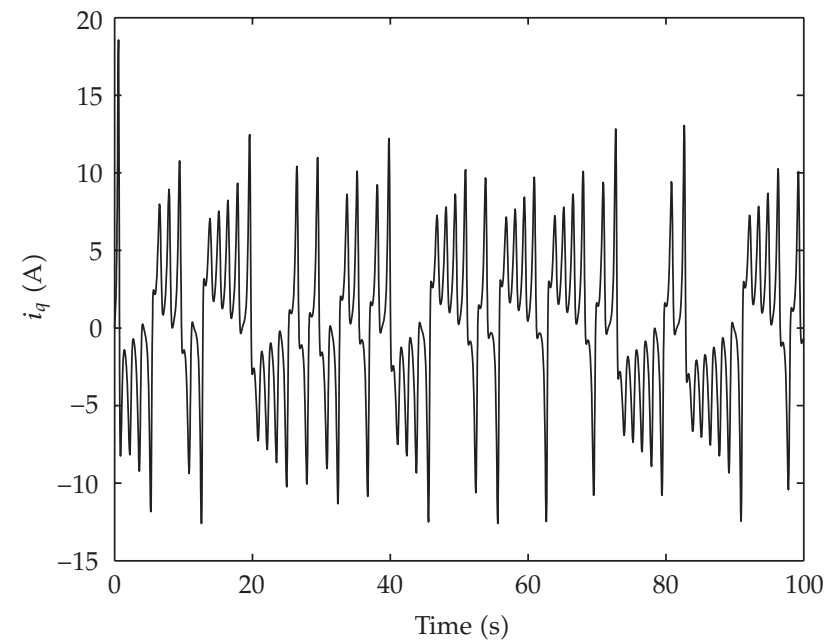

Figure 4: The $i_{q}$ curve of the chaotic PMSM drive system without $u_{d}$.

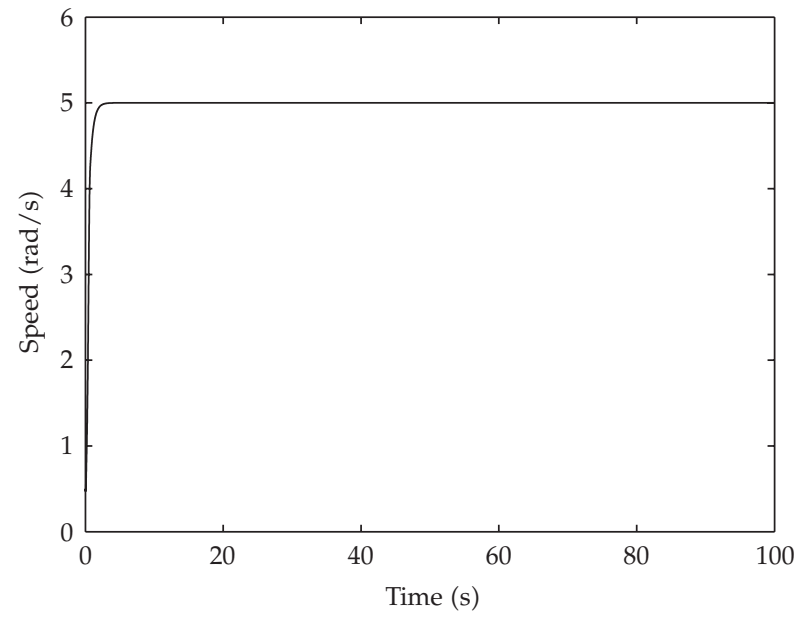

Figure 5: The $\omega$ curve of the chaotic PMSM drive system with $u_{d}$.

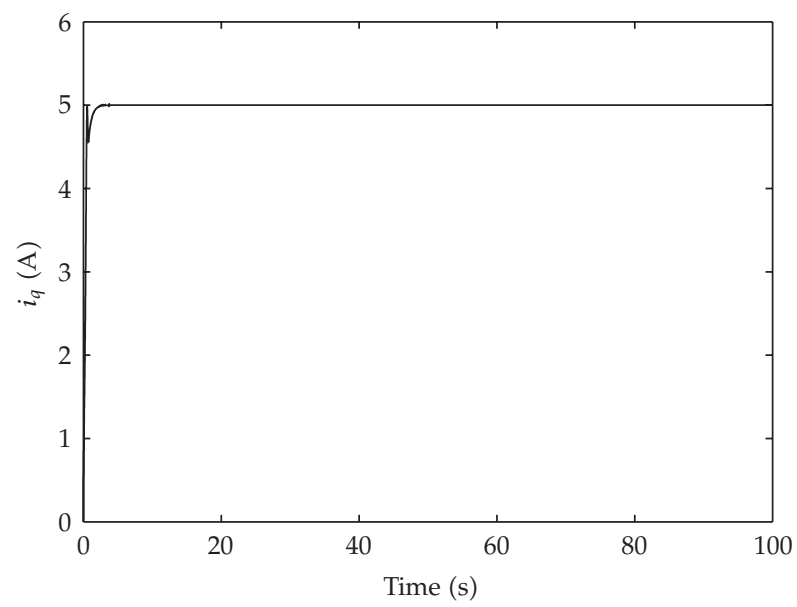

Figure 6: The $i_{q}$ curve of the chaotic PMSM drive system with $u_{d}$. 


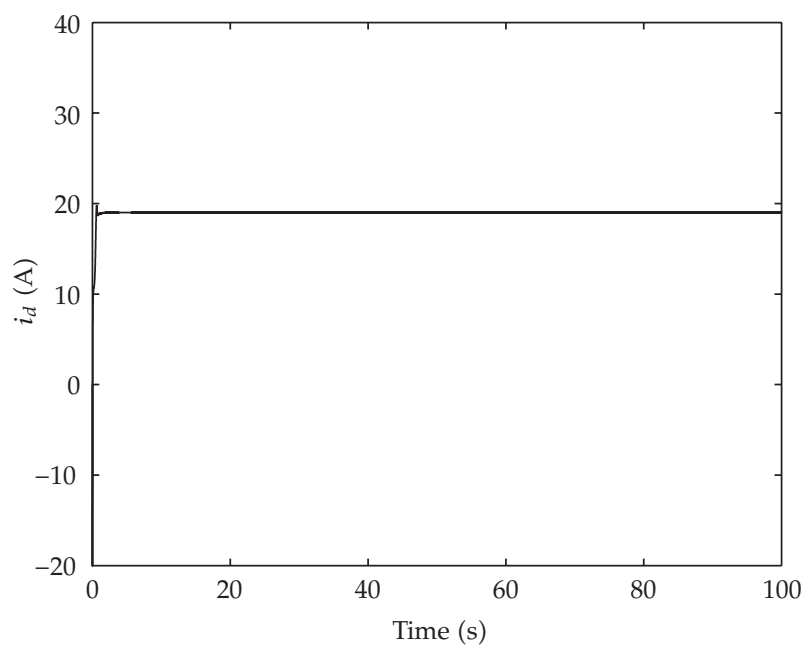

Figure 7: The $i_{d}$ curve of the chaotic PMSM drive system with $u_{d}$.

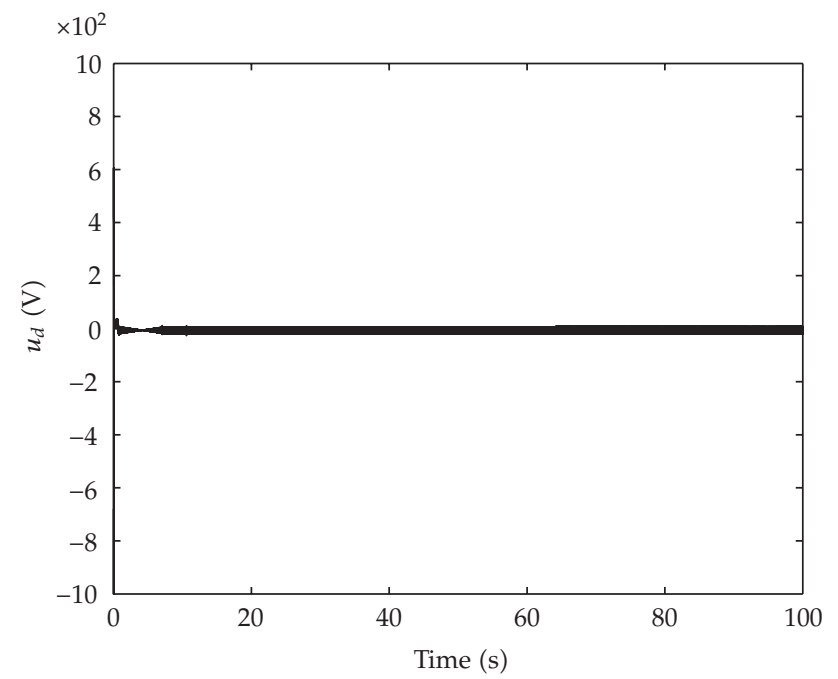

Figure 8: The curve of the controller $u_{d}$.

And the fuzzy membership functions are:

$$
\begin{array}{ll}
\mu_{F_{i}^{1}}=\exp \left[\frac{-(x+5)^{2}}{2}\right], & \mu_{F_{i}^{2}}=\exp \left[\frac{-(x+4)^{2}}{2}\right], \\
\mu_{F_{i}^{3}}=\exp \left[\frac{-(x+3)^{2}}{2}\right], & \mu_{F_{i}^{4}}=\exp \left[\frac{-(x+2)^{2}}{2}\right], \\
\mu_{F_{i}^{5}}=\exp \left[\frac{-(x+1)^{2}}{2}\right], & \mu_{F_{i}^{6}}=\exp \left[\frac{-(x-0)^{2}}{2}\right],
\end{array}
$$




$$
\begin{array}{ll}
\mu_{F_{i}^{7}}=\exp \left[\frac{-(x-1)^{2}}{2}\right], & \mu_{F_{i}^{8}}=\exp \left[\frac{-(x-2)^{2}}{2}\right], \\
\mu_{F_{i}^{9}}=\exp \left[\frac{-(x-3)^{2}}{2}\right], & \mu_{F_{i}^{10}}=\exp \left[\frac{-(x-4)^{2}}{2}\right], \\
\mu_{F_{i}^{11}}=\exp \left[\frac{-(x-5)^{2}}{2}\right] . &
\end{array}
$$

Give the reference signals $x_{d}=5$ and the simulation is carried out for the PMSM drive system. Compared two sets figures, it is seen clearly that the proposed controller can suppress the chaos in PMSM drive system and good tracking performance has been achieved successfully.

\section{Conclusion}

Based on backstepping technique, an adaptive fuzzy control scheme is proposed to control chaos in the permanent magnet synchronous motor drive systems. The proposed controllers guarantee that the tracking error converges to a small neighborhood of the origin, and all the closed-loop signals are bounded. The simulation results are provided to demonstrate the effectiveness and feasibility of the proposed method.

\section{Acknowledgments}

This paper is partially supported by the Natural Science Foundation of China (60674055, $60774027,60774047)$, the Natural Science Foundation of Shandong Province (ZR2009GM034), the State Key Laboratory of Rail Traffic Control and Safety (Beijing Jiaotong University) (RCS2008ZZ004), the cultivating plan of excellent degree thesis (Qingdao University), and Shandong Province Domestic Visitor Foundation.

\section{References}

[1] Z. Li, J. B. Park, Y. H. Joo, B. Zhang, and G. Chen, "Bifurcations and chaos in a permanent-magnet synchronous motor," IEEE Transactions on Circuits and Systems I, vol. 49, no. 3, pp. 383-387, 2002.

[2] U. E. Vincent and A. Ucar, "Synchronization and anti-synchronization of chaos in permanent magnet reluctance machine," Far East Journal of Dynamical Systems, vol. 9, no. 2, pp. 211-221, 2007.

[3] E. Ott, C. Grebogi, and J. A. Yorke, "Controlling chaos," Physical Review Letters, vol. 64, no. 11, pp. 1196-1199, 1990.

[4] H. Ren and D. Liu, "Nonlinear feedback control of chaos in permanent magnet synchronous motor," IEEE Transactions on Circuits and Systems II, vol. 53, no. 1, pp. 45-50, 2006.

[5] Y. Gao and K. T. Chau, "Chaotification of permanent-magnet synchronous motor drives using timedelay feedback," in Proceedings of the 28th Annual Conference of the IEEE Industrial Electronics Society, vol. 2, pp. 762-766, November 2002.

[6] H. Ren, D. Liu, and J. Li, "Delay feedback control of chaos in permanent magnet synchronousmotor," in Proceedings of the China Society Electronic Engineering Conference, pp. 175-178, 2003.

[7] H. Ren and D. Liu, "Nonlinear feedback control of chaos in permanent magnet synchronous motor," IEEE Transactions on Circuits and Systems II, vol. 53, no. 1, pp. 45-50, 2006.

[8] A. M. Harb, "Nonlinear chaos control in a permanent magnet reluctance machine," Chaos, Solitons and Fractals, vol. 19, no. 5, pp. 1217-1224, 2004. 
[9] D. Q. Wei, X. S. Luo, B. H. Wang, and J. Q. Fang, “Robust adaptive dynamic surface control of chaos in permanent magnet synchronous motor," Physics Letters A, vol. 363, no. 1-2, pp. 71-77, 2007.

[10] Z. Li, G. Chen, S. Shi, and C. Han, "Robust adaptive tracking control for a class of uncertain chaotic systems," Physics Letters A, vol. 310, no. 1, pp. 40-43, 2003.

[11] M. Krstic, I. Kanellakopoulus, and P. Kokotovic, Nonlinear and Adaptive Control Design, John Wiley \& Sons, New York, NY, USA, 1995.

[12] A. A. Zaher and M. A. Zohdy, "Robust control of biped robots," in Proceedings of the American Control Conference (ACC '00), pp. 1473-1477, Chicago, Ill, USA, June 2000.

[13] A. Harb and A. Zaher, "Nonlinear recursive chaos control," in Proceedings of the American Control Conference (ACC '00), Anchorage, Alaska, USA, 2002.

[14] A. Harb and W. Ahmad, "Control of chaotic oscillators using a nonlinear recursive back-stepping controller," in Proceedings of the IASTED Conference on Applied Simulations and Modeling, pp. 451-453, Crete, Greece, 2002.

[15] L. A. Zadeh, “Fuzzy sets," Information and Computation, vol. 8, no. 3, pp. 338-353, 1965.

[16] Y. Zheng and G. Chen, "Fuzzy impulsive control of chaotic systems based on TS fuzzy model," Chaos, Solitons and Fractals, vol. 39, no. 4, pp. 2002-2011, 2009.

[17] B. Chen, X. Liu, and S. Tong, "Adaptive fuzzy approach to control unified chaotic systems," Chaos, Solitons and Fractals, vol. 34, no. 4, pp. 1180-1187, 2007.

[18] C. C. Lee, "Fuzzy logic in control systems: fuzzy logic controller. I, II," IEEE Transactions on Systems, Man, and Cybernetics, vol. 20, no. 2, pp. 404-435, 1990.

[19] L. Wang and J. M. Mendel, "Fuzzy basis functions, universal approximation, and orthogonal leastsquares learning," IEEE Transactions on Neural Networks, vol. 3, no. 5, pp. 807-814, 1992. 


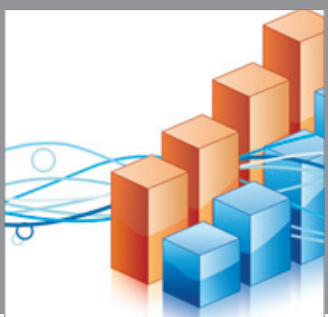

Advances in

Operations Research

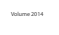

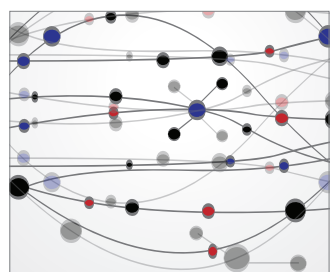

\section{The Scientific} World Journal
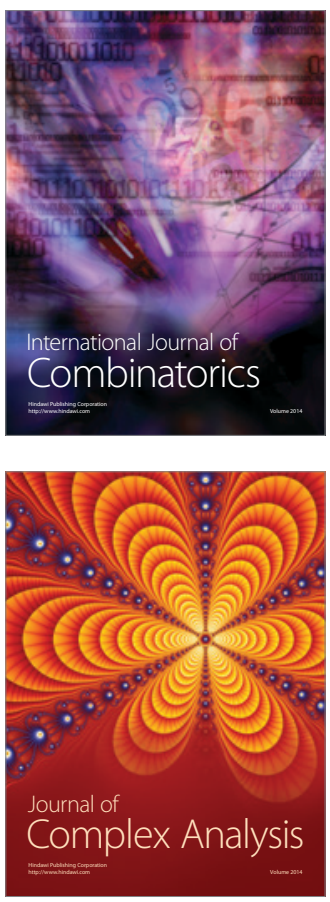

International Journal of

Mathematics and

Mathematical

Sciences
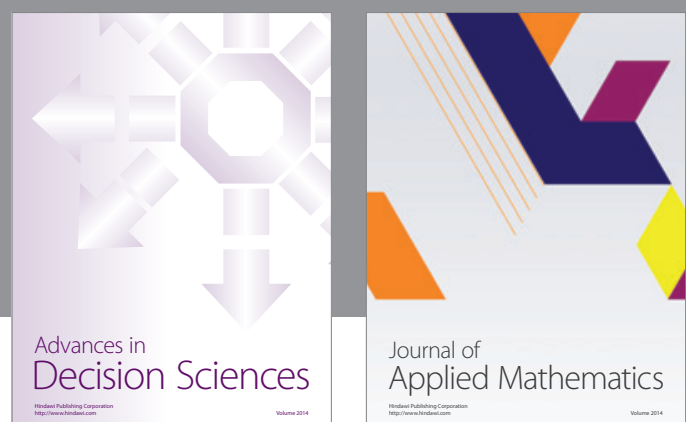

Journal of

Applied Mathematics
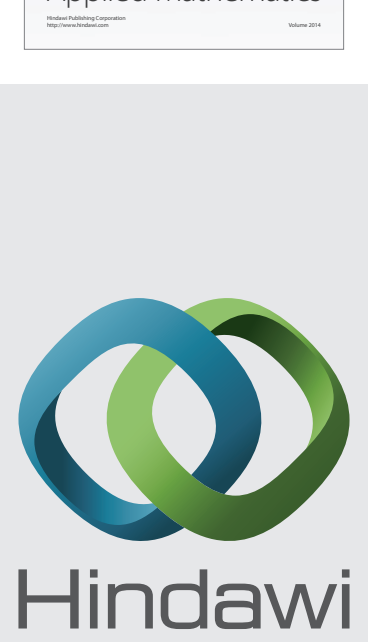

Submit your manuscripts at http://www.hindawi.com
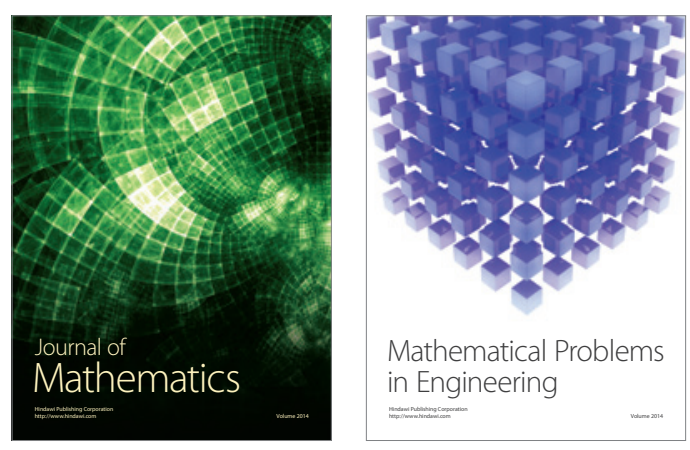

Mathematical Problems in Engineering
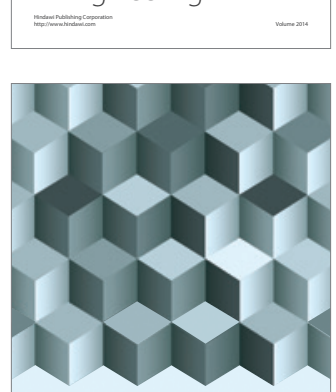

Journal of

Function Spaces
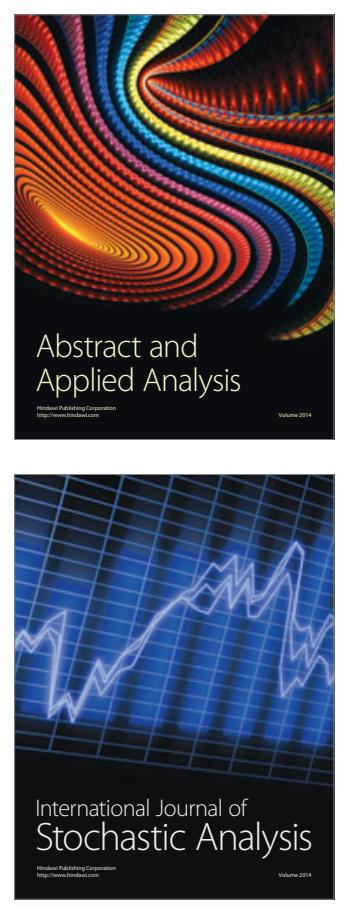

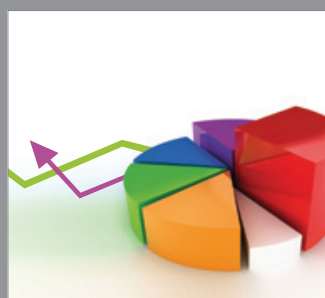

ournal of

Probability and Statistics

Promensencen
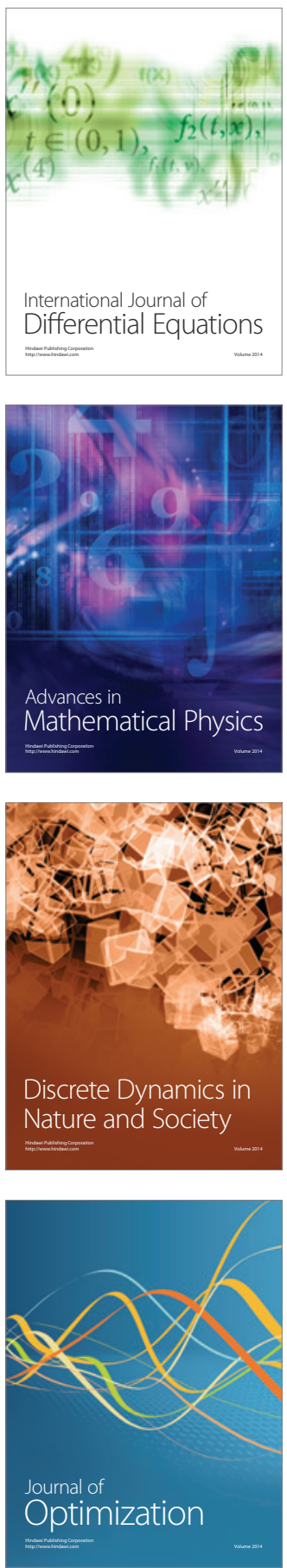